\title{
Three Dimensional Comparison of Interventional MR Radiofrequency Ablation Images with Tissue Response
}

\author{
Michael S. Breen ${ }^{1}$, David L. Wilson ${ }^{1,2}$, Roee S. Lazebnik ${ }^{1,3}$, and \\ Jonathan S. Lewin 1,2 \\ ${ }^{1}$ Department of Biomedical Engineering, Case Western Reserve University \\ $\{d l w, m s b$, rsl\}@po.cwru.edu lewin@uhrad.com \\ 2 Department of Radiology, University Hospitals of Cleveland \\ 3 School of Medicine, Case Western Reserve University, Cleveland, Ohio, USA
}

\begin{abstract}
Solid tumors are being treated using radio-frequency (RF) ablation under interventional magnetic resonance imaging (MRI) guidance. We are investigating the ability of MR to monitor ablation treatments by comparing MR images of thermal lesions to histologically assayed tissue damage. We developed a methodology using a three dimensional computer registration. An open MRI system was used to guide an electrode into five rabbit thighs and acquire MR volumes post ablation. After MR and histology images were registered with an accuracy of $1.32 \pm 0.39 \mathrm{~mm}$ (mean $\pm \mathrm{SD}$ ), a boundary of necrosis identified in histology was compared with the outer boundary of the hyperintense rim in MR images. For 14 T2-weighted MR images, the absolute distance between boundaries was $0.96 \pm 0.34 \mathrm{~mm}$ (mean \pm SD). Since the small discrepancy between boundaries is comparable to our registration accuracy, the boundaries probably match exactly. Similar correlations to histology were obtained with a deformable model segmentation method. This is good evidence that during treatments, MR images can localize the necrotic region.
\end{abstract}

\section{Introduction}

Solid tumors and other pathologies are treated using radio-frequency (RF) thermal ablation under magnetic resonance (MR) guidance. Magnetic resonance imaging (MRI) has several advantages including the lack of ionizing radiation, excellent soft tissue discrimination, sensitivity to blood flow and temperature, and the ability to image at any angle. In addition, MR imaging appears to be more accurate than ultrasound or computed tomography for monitoring the hyperacute effects of thermal ablation treatment [1,2]. The major contribution of MRI is its potential to monitor the zone of thermal tissue destruction during the procedure. To monitor an ablation procedure, MRI can intermittently measure temperature during heating and MR structural images during and after treatment. We investigated over several years this unique ability to monitor treatment by comparing MR lesion images acquired approximately 45 minutes after treatment to tissue damage as seen histologically using three-dimensional (3D) registration techniques. The ability of these MR images to accurately predict the region of eventual cell death would allow complete 
destruction of the disease tissue volume, including the margin, while avoiding the destruction of nearby normal tissue of critical importance.

Being able to determine the region of tissue destruction in live time would be significant. With an assessment of the treatment soon after ablation, one could adjust the therapy to compensate for deviations from preprocedural predictions. One can apply treatment at multiple locations. In addition, RF ablation procedures could be extended to the safe destruction of tumor adjacent to vital structures that might be damaged by heating.

Previous studies indicate that regions of low MR signal in images obtained minutes after thermal ablation correlate with regions of tissue damage [1,3-4]. Analysis was typically done using geometric measurements without alignment of histology to MR images [1,3]. For example, in normal in vivo rabbit liver, Lee et al. [3] manually measured diameters of T2-weighted and gadolinium contrast-enhanced (CE) T1-weighted MR lesion images, and diameters of color changes in sectioned fresh tissue. In six lesions, diameters agreed within $3 \mathrm{~mm}$. The typical response was a central hypointense region surrounded by a hyperintense rim. Lewin et al. [1] manually estimated axial dimensions and used an ellipsoidal model to estimate tumor and thermal lesion volumes in humans with no tissue confirmation. From these studies, there is evidence that MR thermal lesion images reflect tissue damage. However, a careful regional correlation requires the alignment of histology to MR images. Our studies will carefully examine the relationship between cell damage and MR images on a voxel-by-voxel basis.

Our careful analysis of post-ablation MR images with the tissue response has several advantages. First, it could allow for a better understanding of mechanism responsible for the MR signal changes. These MR signals changes could be due to different zones of necrosis or different zones of increased extracellular space. Second, this would allow one to compare the mechanism and pattern of tissue destruction of RF with other ablation techniques such as laser, even though similar MR observations are found. Finally, it could better explain the macroscopic tissue color changes which is used by several investigators to assess tissue damage $[1,3]$.

In this study, we correlated hyperacute T2-weighted in vivo MR images with the tissue response. Using a rabbit thigh muscle model, we performed RF ablations and related MR with histological images using a 3D registration method. Changes in the MR magnitude signals were compared with the histo-pathological observations. We examined the hypothesis that the hyperintense region observed in T2-weighted MR thermal lesion images is a good predictor of eventual cell death. We present several experimental results showing the accuracy of MR images to reflect the tissue response.

\section{Methods}

Following a protocol approved by the Institutional Animal Research Committee, rabbits were anesthetized. After shaving each animal's abdomen and left thigh, we placed the rabbits in the prone position within the gantry of a clinical $0.2 \mathrm{~T}$ C-arm 
MR imaging system (Siemens MAGNETOM OPEN, Erlangen, Germany). The legs of each rabbit were secured to a customized Plexiglas support to prevent movement of the thighs. After positioning two wire mesh grounding pads coated with conductive gel on each rabbit's abdomen, we placed the thighs within a $12 \mathrm{~cm}$ diameter multi-turn solenoid, receive-only coil.

Under MR guidance, we inserted an MR-compatible titanium RF electrode with a $10 \mathrm{~mm}$ exposed tip (Radionics, Burlington, MA) percutaneously into the thigh muscle. The lesion formation was achieved by increasing the local temperature by delivering RF electric current between the electrode tip and ground pads. We applied RF energy for 2 minutes using a $100 \mathrm{~W}$ RF generator operating at $500 \mathrm{kHz}$ (RFG-3C, Radionics, Burlington, MA). The tip of the RF electrode was maintained at a temperature of $90+/-2^{\circ} \mathrm{C}$ using a thermistor within the electrode tip. Immediately after ablation, 2 MR-compatible fiducial needles were inserted into the thigh near the thermal lesion. Approximately 10 minutes after ablation, we acquired MRI volumes of the thigh. A T2-weighted MR sequence was applied to yield $0.70 \times 0.70 \times 3.0-\mathrm{mm}$ voxels oriented to give the highest resolution for slices perpendicular to the RF electrode. Figure 1 shows a typical T2-weighted MR image minutes after ablation. To examine latent tissue changes in response to the interventional procedure, the rabbits were sacrificed approximately 45 minutes after the ablation. After fixation, we removed the thigh from the bone for gross pathological and histological examination.

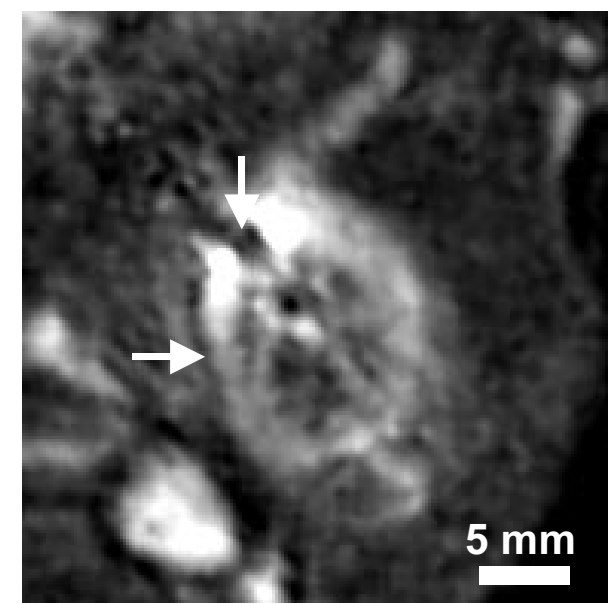

Fig. 1. A typical T2-weighted in vivo MR image acquired minutes after ablation. The thermal lesion (horizontal arrow) is the bright elliptical region that has an isointense/hypointense inner region surrounded by a hyperintense outer region. The small bright dot at the center of the lesion is blood and/or interstitial fluid that filled the track of the RF electrode, which was withdrawn prior to imaging. The small dark region (vertical arrow) is an MR image artifact of a fiducial needle inserted after the ablation, which was used to align the MR and tissue image volumes. 
We obtained $3 \mathrm{~mm}$ tissue slices by using a specially designed apparatus that included a tissue platform, digital camera, and a linear displacement device for accurate stepping of the platform in small increments. To orient the specimen and reduce deformation during slicing, we embedded the specimen in tissue embedding wax. Tissue slicing was done in planes approximately perpendicular to a fiducial needle. We sliced the specimen with a tissue-slicing knife using vertical supports of the apparatus as a guide. Repeatedly, we photographed the tissue block face, advanced the platform by $3 \mathrm{~mm}$, and sliced the tissue, until the specimen was traversed. The tips of the fiducial needles were exposed at the tissue block face for easy identification in each photograph, and stepped back slightly beyond the plane of the next tissue slice. We calibrated these macroscopic tissue images using a ruler in the plane of the tissue slice. The tissue images yield square pixels that were typically $0.17 \mathrm{~mm}$ on a side. We embedded the tissue in paraffin and obtained hematoxylin and eosin (H\&E) and Masson trichrome (MT) stained histological samples.

Histology slides were digitized using a video microcopy system that consisted of a light microscope, video camera, position encoded motorized stage, and controller software. To obtain an image of the entire slide, we used a software function that formed a large tiled map image with pixels that were typically $5.21 \mathrm{um}$ on a side. Using the video microscopy system and software program, we determined the location of tissue damage boundaries on the slide, and marked the appropriate position on the map image. Cellular damage was defined by a loss of muscle's naturally occurring birefringence, which has been shown to be a reliable indicator of irreversible cell damage [5]. Birefringence properties were determined by examining the slide under polarized light. A distinct boundary was marked, one where the tissue showed a loss of birefringence. The end result of this marking process was a histology image with a cell damage boundary that can be compared with lesion boundaries seen in MR images.

We previously developed a method using 3D registration to align the histological and in vivo MR image data [6]. Briefly, we used the macroscopic tissue images as the reference and registered histology and MR images to them using two different computer alignment steps. First, the MR volume was aligned to the volume of tissue images by registering the fiducial needles placed near the lesion. Second, we registered the histology images with the tissue images using a $2 \mathrm{D}$ warping technique that aligned internal features and the outside boundary of histology and tissue images. The above steps allowed us to match a pixel in a histology image with an interpolated sample from the in vivo MR image volume. This registration method was previously validated by Breen et. al. [6], and the accuracy determined from displacement of needle fiducials was estimated to be $1.32 \mathrm{~mm} \pm 0.39 \mathrm{~mm}$ (mean $\pm \mathrm{SD}$ ).

We previously created a twelve-parameter, three-dimensional, globally deformable model with quadric surfaces that describes lesion boundaries [7]. The model was automatically fitted to the grayscale MR volume through minimization of an objective function that simultaneously considers both $3 \mathrm{D}$ lesion geometry and $3 \mathrm{D}$ grayscale boundaries. The method attempts to place the inner and outer surfaces such that they intersect the greatest average dark-to-light and light-to-dark transitions 
respectively. We applied this method to the T2-weighted MR images, and compared results to manually segmented $\mathrm{MR}$ and $3 \mathrm{D}$ registered histological boundaries of cellular damage.

To evaluate the ability of MR images to predict tissue response, we compared the tissue damage boundary identified in histology with boundaries marked by an observer in MR images. The observer manually segmented the outer boundary of the hyperintense region in the T2-weighted MR images. Segmentation was performed using a freehand region of interest tool in Analyze, an image analysis software. For each tissue slice, an automatic algorithm determined equally spaced points, $0.25 \mathrm{~mm}$ apart, along a spline interpolated along the histology boundary. For each such point, the algorithm found the closest point along a continuous spline interpolated from the corresponding MR boundary. A signed 2D Euclidean distance between each point pair was determined such that if the MR point is closer to the boundary centroid than the histology point, the distance is negative, else it is positive. This allowed us to determine if one boundary was interior or exterior to the other.

\section{Results}

In Figure 2, we copied the boundary of cell necrosis identified manually in the histological samples to the registered MR images. In this necrotic region, the cells were characterized by shrinkage or loss of nuclei, shrunken and distorted with a wavy or fragmented appearance, and associated with marked interstitial edema. The cytoplasm showed evidence of contraction band necrosis and a loss of muscle's birefringence. This cell damage boundary matched closely to the outer boundary of the hyperintense region in MR images. In some slices, histology sections were intact over the entire lesion; here we show a histology fragment that again was registered using the $3 \mathrm{D}$ registration steps. This fragment was chosen because of the clear staining. By comparing the two images in the right column, one can see that many small features in the histology boundary, particularly along the right-most edge, are present in the MR images. Results were remarkably similar across several adjoining images.

To further investigate the correlation of MR and histology images, we compared the necrotic boundary with the outer boundary of the hyperintense region marked by an observer in the T2-weighted MR images. For five rabbit experiments, the boundaries were well aligned in 14 registered histology slices with an absolute distance of $0.96 \pm 0.34 \mathrm{~mm}$ (mean $\pm \mathrm{SD}$ ). The signed distances were not significantly different from zero $(\mathrm{P}=0.142)$, which is good evidence of insignificant bias between the boundaries. Once again, 3D registration was independently achieved using fiducial needles. Furthermore, we compared manually segmented histological sections with registered automatically segmented MR images. The automated and histology boundaries matched closely in all registered histology slices for both the inner and outer boundary. 

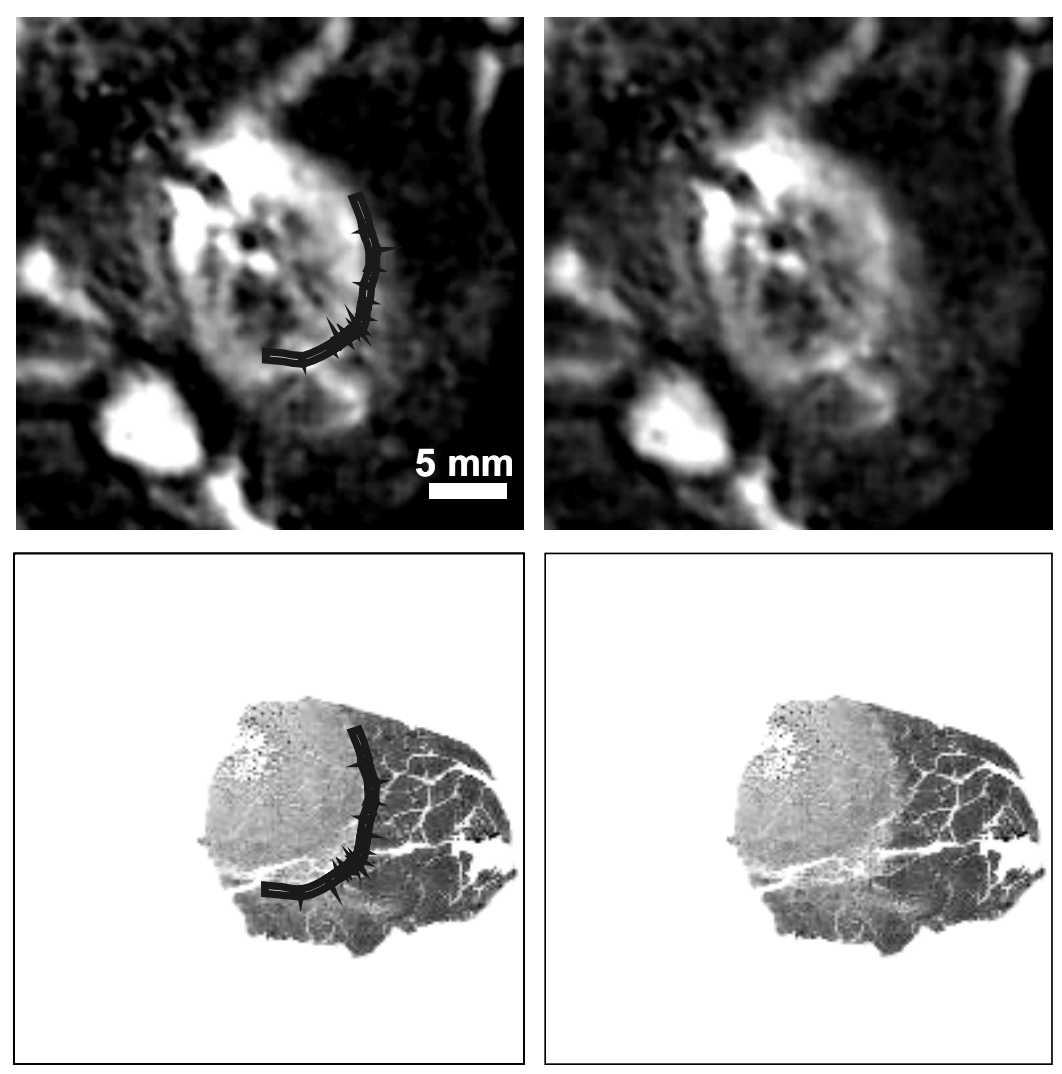

Fig. 2. Comparison of tissue damage boundary identified in histology with MR image features. Registered images on the left and right are identical except for graphical overlays on the left showing the thermal damage boundary. Images are: in vivo T2-weighted MR images (top), and Masson tricrhrome stained histology images (bottom). The boundary corresponds to a region of decreased stain uptake and a loss of the muscle's birefringence. Boundaries are marked on histology image with graphical overlays and copied to registered MR images, where they match features in these images. Other bright regions in the MR images are streaks of fat and the elliptical

\section{Discussion}

Our results suggest that it is possible for hyperacute (within minutes) MR lesion image to predict the tissue response. Features such as $3 \mathrm{D}$ registration of in vivo MR images to histology images, and accurate segmentation of tissue damage boundaries on a large histology image are important steps to accurately correlate the tissue response to in vivo MR thermal lesions images. For these experiments, we determined that the outer boundary of the hyperintense region in MR images and boundary of necrosis in histology are well correlated. The mean difference between the boundaries 
for T2-weighted images was $0.96 \mathrm{~mm}$. This value compares favorably to the MR in plane voxel width $(0.70 \mathrm{~mm})$ and thickness $(3.00 \mathrm{~mm})$.

From histology images, there was a distinct central region characterized by muscle cells with total loss of birefringence and contraction band necrosis. Surrounding this was an exceptionally well demarcated peripheral region having increased extracellular spaces, and cells with damaged of contractile elements, partial loss of birefringence, and contraction band necrosis. This tissue response was very sharply delimited against adjacent normal tissue. It is believed that the central and peripheral regions correspond to the region of eventual necrosis.

Although the exact determination of cell viability is difficult, proliferative contraction band necrosis within the region of birefringence loss suggest it is likely non-viable. Upon thermal injury, a loss of birefringence occurs due to the disarray of the regular matrix of the actin and myosin molecules, and this has been previously shown to correspond to a region of eventual necrosis [5].

A comparison of MR and tissue response 45 minutes post-ablation showed that outer boundary of hyperintense region in MR images closely corresponds to the region of dead or irreversibly damaged cells in histology. These results are consistent with our findings from experiments with rabbits sacrificed four days post-ablation, which show the complete extent of the cell death in a well demarcated region of necrosis in histology. A correlation of histology and MR images four days post-ablation showed the boundary of cell death closely corresponds to the outer boundary of the hyperintense region with a mean absolute distance for 20 tissue slices of $0.95 \pm 0.34 \mathrm{~mm}$ for T2-wieghted MR images. To relate the outer boundary of the hyperintense region in MR images four days and 45 minutes post-ablation, we developed a 3D ellipsoidal model that is described in detail and validated elsewhere [7]. We fitted this model to the outer boundary of the hyperintense region in both MR volumes for 10 lesions. The longest radius of the ellipsoid corresponds to the direction of the RF electrode. Since we slice our tissue approximately perpendicular to the RF electrode, we compared the two short radii of the ellipsoid at 45 minutes and four days post-ablation. At 45 minutes post-ablation, the mean length of the two short radii were not statistically different, indicating a circularly symmetric lesion along the RF electrode. Similar results were obtained at four days post-ablation. Furthermore, the mean length of the two short radii at 45 minutes and four days post-ablation were not statistically different. The mean length of the longest radius increased by typically $3.5 \mathrm{~mm}$ between 45 minutes and four days post-ablation. Therefore, we can infer that the outer boundary of the hyperintense region in the MR images 45 minutes post-ablation is a reliable marker of cell death with no evidence of cell recovery.

It is possible that the MR and histology boundaries match exactly and the small measured differences are due to limitations of our methodology, for a variety of reasons. First, registration error might arise from both the alignment of the MR volume with the tissue sections and the warping of histological sections to the tissue sections. Second, we note that although registration was performed in $3 \mathrm{D}$, distance calculations were performed on a $2 \mathrm{D}$ basis due to the slice nature of histological sections. This approach tends to overestimate distance errors as for a given point 
along a histology border, the closest point along the MR boundary may be slightly out of plane. Third, the in-plane resolution of the histological images $(0.17 \mathrm{~mm}$ pixel width) was approximately four times that of the acquired MR slices $(0.7 \mathrm{~mm}$ voxel width). This allows partial volume effects to blur several histological pixels into a single MR voxel. In addition, the 3D registration and re-slicing procedure may further reduce the effective in-plane MR resolution through out-of-plane tilting, as the MR slice thickness was greater than in-plane resolution. However, this effect was minimized by acquiring both histological and MR data approximately perpendicular to the electrode path. Overall, given the above considerations, the small detected differences between corresponding histology and MR boundaries are likely insignificant; the outer boundary of the hyperintense region in MR probably corresponds to cell death.

We conclude that our 3D methodology can be used to accurately map tissue response to MR thermal lesion images. Results show that in the rabbit thigh muscle, the outer boundary of the hyperintense region in T2-weighted MR images obtained minutes after RF ablation closely corresponds to the region of cell damage. This is good evidence that iMRI thermal lesion images can be used for real-time feedback during thermal RF ablation treatments.

Acknowledgments. This research was supported by NIH grant R01-CA84433 and R01-CA81431. MSB was supported, in part, by an NIH training grant. RSL was supported by a Whitaker Foundation graduate fellowship and the CWRU Medical Scientist Training Program.

\section{References}

1. J.S. Lewin, C.F. Connell, J.L. Duerk, "Interactive MRI-guided radiofrequency interstitial ablation of abdominal tumor: Clinical safety and feasibility," JMRI, vol. 8, pp. 40-47, 1998.

2. G.S. Gazelle, S.N. Goldberg, L. Solbati, T. Livraghi, "Tumor ablation with radiofrequency energy," Radiology, vol. 217, pp. 633-646, 2000.

3. J.D. Lee, J.M. Lee, S.W. Kim, C.S. Kim, W.S. Mun, "MR imaging-histopathologic correlation of radiofrequency thermal ablation lesion in a rabbit liver model: observation during acute and chronic stages," Korean Journal of Radiology, vol. 2, pp. 151-158, 2001.

4. P.R. Morrison, F.A. Jolesz, D. Charous, et.al., "MRI of laser-induced interstitial thermal injury in an in vivo animal liver model with histologic correlation," JMRI, vol. 8, no. 1, pp. 57-63, 1998.

5. S. Thomsen, J.A. Pearce, W.F. Cheong, "Changes in birefringence as markers of thermal damage in tissues," IEEE Transactions on Biomedical Engineering, vol. 36, no. 12, pp. 1174-1179, 1989.

6. M.S. Breen, T.L. Lancaster, R.S. Lazebnik, S.G. Nour., J.S. Lewin, D.L. Wilson, “Three dimensional method for comparing in vivo interventional MR images of thermally ablated tissue with tissue response," JMRI, vol. 18, pp. 90-102, 2003.

7. R.S. Lazebnik, B.D. Weinberg, M.S. Breen, J.S. Lewin, D.L. Wilson, "Automatic parametric model-based 3D lesion segmentation of MR-guided radiofrequency ablation therapy," IEEE Transactions on Medical Imaging; Submitted. 